\title{
Effective Range Corrections in Few-Body Systems with large Scattering Length
}

\section{Platter}

Department of Physics \& Astronomy, Ohio University, Athens, OH 45701, U.S.A.

The effective field theory with contact interactions alone is a powerful tool to compute low-energy observables for three-body systems with large scattering length. Recent calculations including effective range corrections are discussed and results are presented.

\section{Introduction}

Effective field theories (EFT) exploit an existing separation of scales and describe physical systems with a minimal set of degrees of freedom. In the last years the effective field theory with contact interactions alone (CEFT) has been applied very successfully to various few-body systems with large scattering length [ 1, 2, 3]. Furthermore, recent publications concentrated on the inclusion of higher order corrections in calculations for three-body observables [4, 5]. However, an obvious problem within this framework, is the question on the power counting of many-body forces. While a consistent power counting has been used in previous calculations for the three-nucleon system with great success [ 4], we have shown recently [6] that an energy-dependent three-body force can be shifted down by one order when using a subtraction formalism to compute observables. This allows to analyze two-parameter correlation plots to one order higher than previously thought and improves the predictive power of the CEFT.

In the following two sections we will report on recent results obtained within this framework. The last section summarizes our findings and gives an outlook for further possible calculations.

\section{The Three-Boson System}

The CEFT is formulated in terms of the available degrees of freedom, i.e. heavy boson or fermion fields only and is valid if the underlying potential is short-ranged and the involved momentum is smaller than the inverse range of the potential. The most general Lagrangian describing non-relativistic bosons interacting through contact interactions only is given by

$\mathcal{L}=\psi^{\dagger}\left[i \partial_{t}+\frac{\vec{\nabla}^{2}}{2 M}\right] \psi-\frac{C_{0}}{2}\left(\psi^{\dagger} \psi\right)^{2}-\frac{D_{0}}{6}\left(\psi^{\dagger} \psi\right)^{3}+\ldots$ 

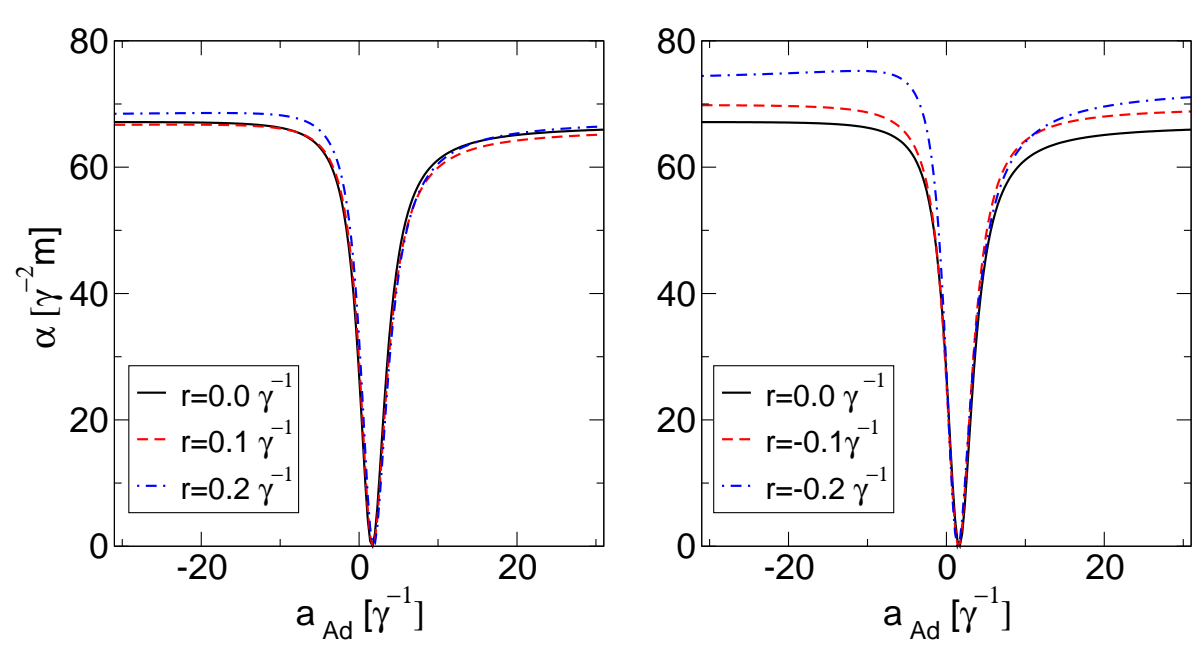

Figure 1. The left and right plot show the recombination coefficient $\alpha$ plotted against the atom-dimer scattering at NLO. In the left plot results are plotted for positive effective ranges $r=0.0,0.1 \gamma^{-1}$ and $0.2 \gamma^{-1}$ (solid, dashed and dot-dashed line, respectively). In the right plot results are plotted for negative effective ranges $r=0.0,-0.1 \gamma^{-1}$ and $-0.2 \gamma^{-1}$ (solid, dashed and dot-dashed line, respectively).

where the ellipses denote interactions with more derivatives and/or more fields. With the corresponding power counting this EFT is an expansion in $R / a$, where $R$ denotes the range of the underlying potential and $a$ the two-body scattering length. It is a particular feature of this theory that when applied to the the three-body sector, a three-body force is needed already at leading order [1]. However, instead of using an explicit three-body force one can use alternatively a subtraction formalism which trades the three-body force in the kernel of the three-body Faddeev equation for an explicit three-body observables in the inhomogenity of the integral equation [ 7, 8]. It was shown recently that when this framework is used an energy dependent three-body force needs not to be included at next-to-next-to-leading order (NNLO) [ 6 .

This allows in particular to describe observables of ${ }^{4} \mathrm{He}$ few-body systems to very high precision. Since the atom-atom scattering length is $a_{2} \sim 100 \AA$ while the typical van der Waal's forces between the Helium atoms have a range $R \approx 10 \AA$, the EFT expansion parameter is $R / a_{2} \approx 0.1$ and the EFT expansion should converge very quickly. In absence of any experimental information about the binding energies of ${ }^{4} \mathrm{He}$ trimers we use theoretical few-body calculations using "realistic" atom-atom potentials [10, 11, 12, 13, to fix our three-body input and for comparison with our results. Therefore, when using $a_{A d}=1.205 \gamma^{-1}$ as obtained by Roudnev for the TTY potential we obtain $B_{3}^{(0)}=89.38 B_{2}$ for the ground state and $B_{3}^{(1)}=1.737 B_{2}$ for the excited trimer state at NNLO. This is in very good agreement with Roudnev's results for the ground and excited state, $B_{3}^{(0)}=96.33 B_{2}$ and $B_{3}^{(1)}=1.738 B_{2}$, respectively. A further process one can consider within this framework is a common reason for atom losses in experiments with ultracold alkali gases; namely the recombination of three atoms into a shallow dimer and an atom. 


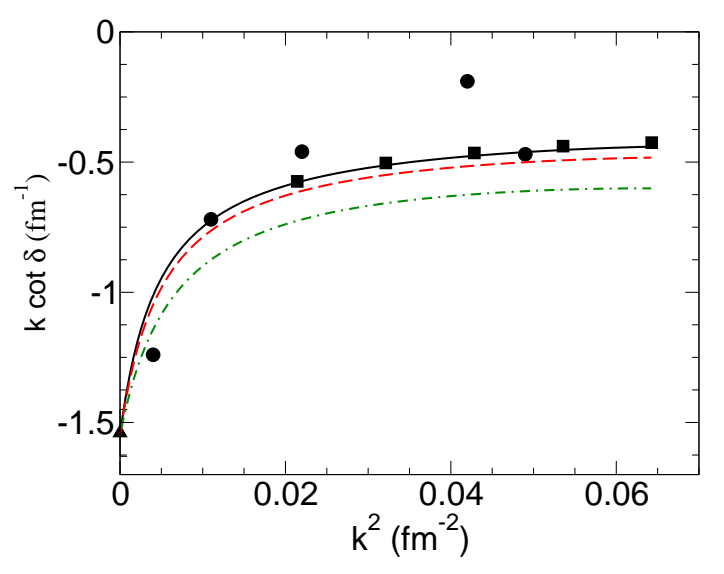

Figure 2. Phase shifts for neutron-deuteron scattering below the deuteron breakup at LO (dashed-dotted line), NLO (dashed line) and NNLO (solid line). The triangle is the result of the scattering length measurement of [17]. The circles are the results of the van Oers-Seagrave phaseshift analysis [18], and the squares denote a phaseshift calculation using a realistic nucleon-nucleon potential [19]

At leading order (LO) the recombination rate has been calculated by Braaten et al. [14]. In Fig [1 we show our results for the correlation between the atom-dimer recombination rate and the atom-dimer scattering length at next-to-leading order (NLO) [ 16]. In the left graph we display results for different negative values of effective range and in the right graph for different positive values. While all curves show a minimum in the recombination coefficient at approximately $a_{A d} \approx 1.7 \gamma$, the maximum value of $\alpha$ depends strongly on the sign and absolute values of the effective range.

\section{The Three-Nucleon System}

The formalism introduced above can easily be applied to fermions and extended to include spin and isospin degrees of freedom. If this is done one is able to compute threenucleon observables in the triton channel up to NNLO with five parameters [ 9]; the singlet and triplet scattering lengths, the corresponding effective ranges and one threebody parameter. For the triton binding energy we obtain $B_{t}^{(\mathrm{LO})}=8.08 \mathrm{MeV}, B_{t}^{(\mathrm{NLO})}=$ $8.19 \mathrm{MeV}$ at LO and NLO, respectively and $B_{t}^{(\mathrm{NNLO})}=8.54 \mathrm{MeV}$ at NNLO if we match to the neutron-deuteron scattering length $a_{n d}=0.65 \mathrm{fm}$ [17]. We have also calculated the corresponding phaseshifts and our results are shown in Fig 2 , For comparison we show in the same figure the results of a forty year old phaseshift analysis [18] and a theoretical calculation using a realistic nucleon-nucleon potential [19]. At higher order our results seem to describe the experimental data better but considering the age of the analysis and the fact that no errors are given for these data, the theoretical calculation by Kievsky et al. should be considered as the true benchmark test for our calculation. At NLO our results already lie significantly closer to this calculation and nearly perfect agreement is achieved at NNLO. It should be noted that our results at LO and NLO order agree 
with previous EFT calculations results given in [15, 4, 7]. We also achieve qualitative agreement at NNLO with Ref.[4], however, without employing an additional three-body counterterm.

\section{Summary}

The CEFT is designed for the calculation of low-energy observables for systems with short-range interactions. When applied to systems with large scattering length, the EFT expansion parameter is $R / a$. It is universal in the sense that it can be applied to any short-ranged interaction at low enough energies and therefore, it is particular well suited to compute observables in atomic and nuclear systems which have a large two-body scattering length. Here, we have dicussed results for low-energy three-body observables with effective range correction included up to NNLO obtained without including a second three-body datum at this order as dictated by a renormalization group analysis given in [6]. This increases the predictive power at this order as observables are described in terms of one renormalization parameter less as has been done in previous calculations in the CEFT. Our results are showing the expected convergence behavior and agree very well with previous theoretical calculations and experiment. It should be noted that this doesn't indicate that results previously obtained at NNLO are wrong but rather that they can be obtained with less input information. Further effort should be devoted on the calculation of electromagnetic properties with the CEFT [20].

\section{REFERENCES}

1. P. F. Bedaque, H.-W. Hammer, and U. van Kolck, Phys. Rev. Lett. 82, 463 (1999).

2. E. Braaten and H.-W. Hammer, Phys. Rept. 428, 259 (2006).

3. L. Platter, H.-W. Hammer and U.-G. Meißner, Phys. Lett. B 607, 254 (2005).

4. P. F. Bedaque, G. Rupak, H. W. Griesshammer and H. W. Hammer, Nucl. Phys. A 714, 589 (2003).

5. H. W. Griesshammer, Nucl. Phys. A 744, 192 (2004).

6. L. Platter and D. R. Phillips, Few-Body Systems in press.

7. I.R. Afnan and D.R. Phillips, Phys. Rev. C 69, 034010 (2004).

8. H.-W. Hammer and T. Mehen, Nucl. Phys. A 690, 535 (2001).

9. L. Platter, Phys. Rev. C 74, 037001 (2006).

10. V. Roudnev and S. Yakovlev, Chem. Phys. Lett. 328, 97 (2000).

11. V. Roudnev, Chem. Phys. Lett. 367,95 (2003).

12. A.K. Motovilov, W. Sandhas, S.A. Sofianos, and E.A. Kolganova, Eur. Phys. J. D 13, 33 (2001).

13. E. A. Kolganova, A. K. Motovilov and W. Sandhas, Phys. Rev. A 70, 052711 (2004).

14. P. F. Bedaque, E. Braaten and H. W. Hammer, Phys. Rev. Lett. 85, 908 (2000).

15. H.-W. Hammer and T. Mehen, Phys. Lett. B 516, 353 (2001).

16. H. W. Hammer, T. Laehde and L. Platter, in preparation.

17. T. C. Black et al., Phys. Rev. Lett. 90, 192502 (2003); K. Schön et al., Phys. Rev. C 67, 044005 (2003).

18. W. T. H. van Oers and J. D. Seagrave,Phys. Lett. B 21, 526 (1967).

19. A. Kievsky, S. Rosati, W. Tornow and M. Viviani, Nucl. Phys. A 607, 402 (1996). 
20. L. Platter and H. W. Hammer, Nucl. Phys. A 766, 132 (2006). 